\title{
S'expliquer avec le collectif de geste
}

Le cas de deux internes en chirurgie cardiaque

Discussions and explanations using the collection of gestures: case study with two heart surgery interns

Explicarse con el colectivo del gesto : El caso de dos internos de cirugía cardiaca

Jean-Luc Tomás

\section{OpenEdition}

Journals

Édition électronique

URL : http://journals.openedition.org/activites/2014

DOI : 10.4000/activites.2014

ISSN : 1765-2723

Éditeur

ARPACT - Association Recherches et Pratiques sur les ACTivités

Référence électronique

Jean-Luc Tomás, "S'expliquer avec le collectif de geste », Activités [En ligne], 5-2 | octobre 2008, mis en ligne le 15 octobre 2008, consulté le 21 avril 2019. URL : http://journals.openedition.org/ activites/2014; DOI : 10.4000/activites.2014

\section{(c) (i) (9)}

Activités est mis à disposition selon les termes de la licence Creative Commons Attribution - Pas d'Utilisation Commerciale - Pas de Modification 4.0 International. 


\title{
S'expliquer avec le collectif de geste: le cas de deux internes en chirurgie cardiaque ${ }^{1}$
}

\author{
Jean-Luc Tomás \\ Centre de Recherche sur le Travail et le Développement, EA 4132 \\ Équipe clinique de l'activité, CNAM, 41, rue Gay-Lussac, 75005 Paris \\ jean-luc.tomas@cnam.fr
}

\begin{abstract}
Discussions and explanations using the collection of gestures: case study with two heart surgery interns. This aim of this paper is to discuss how producing professional gestures can be supported by the history of a profession and by the different ways of doing, which are usually carried out within the professional setting. The study, run in a heart surgery department, has enabled us to capture one of the functions belonging to this collective history, and the evaluation of the gestures made by the workers: a resource for individual action. The methodological and theoretical framework used is that of the clinic of activity. We went ahead and filmed the activity of two voluntary interns in their working environment during a surgical operation. These films were followed by self-confrontation, both simple and crossed. Based on two extracts from these interviews, we have attempted to identify the individual resources facilitating the backup for the dialogical activity leading to a precise gesture during work. At the same time, we questioned the psychological function of the collective group regarding professional controversies brought on by a crossed self-confrontation. Moreover, we have tried to differentiate a shared gesture from a « collective of gestures » as an help for a dialogical activity .
\end{abstract}

\section{KEYWORDS}

Gesture, Collective gesture, Collection of gestures, Crossed selfconfrontation, Thought activity, Development

Dans un service de chirurgie cardiaque ${ }^{2}$, le travail des internes ${ }^{3}$ est sous la responsabilité de l'opérateur principal (praticien hospitalier ${ }^{4}$ ou chef de clinique ${ }^{5}$ ). Le plus souvent, c'est le chef de clinique qui est responsable du début de l'intervention. Le praticien hospitalier ne commence son travail d'opérateur qu'après l'incision et l'ouverture du sternum à la scie. Il " monte » sur le champ en casaque stérile pour mettre en place et superviser la circulation extra-corporelle. À la suite de ce temps opératoire, le cœur est arrêté, et l'intervention se poursuit en présence des trois chirurgiens (dans l'ordre croissant des statuts : l'interne, le chef de clinique et le praticien hospitalier). Qu'il s'agisse de pontages ou de remplacement d'une valve, les procédures s'articulent selon des schémas prévus à l'avance. La disposition et les responsabilités des différents professionnels dans le cours de l'intervention se répètent. Concrètement, la fonction des différents professionnels présents sur le champ opératoire est la suivante: le praticien hospitalier est le premier opérateur; le chef de clinique est le premier aide; l'interne est le second aide; l'instrumentiste présente les instruments nécessaires à la

1. Cet article n'aurait pas vu le jour sans l'Observatoire National de la Démographie des Professions de Santé (ONDPS), organisme à l'origine de l'appel d'offre auquel l'Équipe Clinique de l'Activité a répondu.

2. La chirurgie cardiaque est spécifique à plus d'un titre. Nous présentons deux spécificités: i) si le cœur est arrêté, elle nécessite la présence d'un perfusionniste, c'est-à-dire d'un professionnel dont la fonction est de s'occuper de la machine prenant en charge les fonctions de circulation et de respiration du patient; ii) par ailleurs, lors de la plupart des interventions, un(e) instrumentiste est présent sur le champ, ce qui n'est pas le cas pour d'autres chirurgies.

3. L'internat est composé de stages semestriels dans des services agréés.

4. Chirurgien ayant réussi le concours de praticien hospitalier.

5. Chirurgien en stage de post-internat. 
bonne réalisation des gestes opératoires.

Pour ancrer notre propos, nous souhaitons présenter une situation singulière de travail. Il s'agit d'une intervention chirurgicale durant laquelle l'équipe travaille au remplacement d'une valve aortique. L'opération suit un cours sans difficultés particulières. Cependant, à un moment donné, le praticien hospitalier ${ }^{6}$ ote fermement un instrument des mains de l'interne. Alors que ce dernier aspire l'eau mêlée de sang de la cavité du ventricule gauche, le « patron » saisit la canule de l'aspirateur pour reprendre la main sur l'instrument.

Nous souhaitons nous arrêter dans cet article sur cette situation moins pour reprendre le geste du praticien hospitalier que pour discuter de la manière dont les internes l'interprètent. Dans le cadre de notre travail ${ }^{7}$, nous avons procédé à une co-analyse de l'activité chirurgicale de deux internes en situation d'intervention sur le champ opératoire. Les controverses entre ces deux professionnelles, engendrées par l'analyse, nous permettrons d'instruire un domaine déjà étudié par ailleurs: la fonction psychologique du collectif. De notre côté, nous condenserons notre attention sur la fonction psychologique du collectif dans le cours des controverses professionnelles en autoconfrontation croisée $^{8}$. Plus particulièrement, nous tenterons d'expliquer et de comprendre comment l'analyse des gestes, par les internes eux-mêmes, est soutenue ou non par l'histoire collective du métier de chirurgien cardiaque.

\section{1.- La reprise en main d'un geste professionnel}

Détaillons la situation. Le patient est allongé sur la table d'opération. À l'exception de la brèche opératoire, il est couvert par des champs stériles. À sa droite, le praticien hospitalier tente délicatement de disséquer l'anneau aortique avec des instruments spécifiques. L'intervention a débuté depuis deux heures. Après avoir été techniqué ${ }^{9}$ et anesthésié, le patient a subi plusieurs actes opératoires : le sternum a été scié, l'écarteur placé, le péricarde découpé, et l'aorte incisée. Lors du temps opératoire correspondant à l'ablation de la valve aortique, tous les professionnels de la salle sont attentifs à l'évolution de l'opération. L'infirmière circulante est vigilante aux éventuelles demandes de l'instrumentiste, le perfusionniste contrôle les paramètres de la circulation extra-corporelle, l'infirmier anesthésiste et le médecin anesthésiste surveillent les variables physiologiques. Pendant que le praticien hospitalier s'affère à exciser la valve, le chef de clinique, à sa gauche, observe les gestes réalisés sur le champ, veille à ceux effectués par l'interne, fait signe de la tête à l'instrumentiste, relaie les éventuelles consignes du " patron », contrôle les paramètres vitaux du patient affichés sur les moniteurs. Son apparente inactivité s'oppose aux tentatives d'action de l'interne ${ }^{10}$. Ce dernier qui fait face du praticien hospitalier, tient la canule de l'aspirateur avec sa main droite. Sa main gauche est posée sur le champ opératoire près du bord de l'écarteur. La canule de l'aspirateur lui permet de nettoyer le ventricule gauche du patient. Il retire, de la partie opérée, le sang qui viendrait troubler le travail du chirurgien. Dans le même temps, il ôte les débris et les fragments calciques qui ne doivent absolument pas se retrouver dans la circulation sanguine. À gauche de l'interne, l'instrumentiste très expérimentée et préoccupée par le bon déroulement de l'intervention, tient dans sa main droite une coupelle, et avec sa main gauche, elle tend les instruments au praticien hospitalier. La coupelle, remplie d'eau, sert de réceptacle aux morceaux de l'anneau aortique disséqué par le praticien hospitalier.

6. Le praticien hospitalier (PH) en question est aussi Professeur des Universités (soit PUPH) et chef du service. Dans le but de simplifier la lecture, nous le nommerons: « praticien hospitalier », « patron » ou « senior ».

7. Il s'agit d'une étude commanditée par le Ministère de la Santé ayant pour objet la transmission des gestes professionnels en salle d'opération.

8. Méthode utilisée dans les études et les interventions en clinique de l'activité (Clot, Faïta, Fernandez, \& Scheller, 2001).

9. Le terme « techniquer » est utilisé pour rendre compte du travail réalisé par l'infirmier(e) anesthésiste et le médecin anesthésiste avant l'intervention des chirurgiens.

10. Il nous semble important d'insister sur un point: l'interne est expérimenté, il est en dernière année d'internat, et il connaît le lieu de sa nouvelle affectation dans un service de chirurgie cardiaque à la fonction de chef de clinique. 
Épisodiquement, elle guide l'interne dans l'utilisation de l'aspirateur, et elle lui indique de l'index la partie du ventricule gauche à nettoyer.

Puis, à la suite d'un ordre à peine perceptible du praticien hospitalier, l'instrumentiste retire la coupelle, et verse, avec sa main droite, le contenu d'une large coupelle d'eau stérile dans le ventricule gauche.

Pendant que l'instrumentiste inonde le ventricule, le praticien hospitalier adresse à l'interne une consigne: « l'aspiration, vas-y ». Il s'agit en fait de rincer la cavité dans laquelle des débris auraient pu tomber. À la suite de la consigne du praticien hospitalier, le chef de clinique reprend l'instruction. Il dit en s'adressant à l'interne: « vas-y, bien dans le ». Simultanément, il lui montre le geste qu'il doit réaliser en simulant la manière de faire attendue par le "patron ». Avec son bras gauche, jusqu'alors posé sur les champs stériles, il déplace son bras en direction du champ opératoire: index en avant, il mime le geste rapide et énergique d'aspiration que doit réaliser l'interne. Cependant, le geste quelque peu emprunté de l'interne ne satisfaisant pas le praticien hospitalier, celui-ci lui prend vigoureusement l'aspirateur de sa main gauche. Pendant que le «patron » exécute le geste requis, le chef de clinique qui avait replié son bras sur les champs, réitère un geste avec sa main gauche. Il simule à nouveau le geste qu'attend le praticien hospitalier: un geste résolu du poignet. L'interne, qui n'a plus d'instrument dans les mains, observe la situation en se tenant prêt à reprendre l'aspirateur. Cette séquence de travail, qui commence à partir du moment où l'instrumentiste verse l'eau jusqu'à la position d'attente de l'interne décrite ci-dessus, est très courte, elle ne dure pas plus de 5 secondes.

Cependant, ces quelques secondes ont longuement mobilisé deux internes lorsqu'ils ont été confrontés aux images de cette activité.

En effet, dans le cadre de notre étude, nous avons utilisé des enregistrements filmés de l'activité de travail des internes. Les internes ont pu revenir sur les traces vidéo de leur activité à deux reprises. Nous leur avons demandé de se confronter une première fois à leurs images et de les commenter, lors de ce que nous appelons une " autoconfrontation simple". Dans un second temps, associé à un pair, qui a lui-même été filmé et qui a aussi commenté les images de sa propre activité, ils commentent, en notre présence, les images de leur collègue. Lors de cette " autoconfrontation croisée 》 (Clot, et al. 2001), nous tentons de provoquer, à partir des détails de l'activité réalisée et enregistrée, des controverses professionnelles.

L'interne engagé dans la situation que nous avons décrite a ainsi commenté son activité en autoconfrontations simple, puis associé à un pair ${ }^{11}$, en autoconfrontation croisée.

\section{2.- C'est seulement en mouvement que l'activité montre ce qu'elle est}

Le titre de ce paragraphe reprend, en la modifiant, la phrase de Vygotski (1931/1978): « c'est seulement en mouvement qu'un corps montre ce qu'il est » (p. 65). De notre côté, nous pensons que la méthodologie historico-développementale (Clot, 2004), dont l'un des supports est les images de l'activité des professionnels concernés, leur permet de prendre et reprendre en main, ou non, les situations de travail qu'ils traversent. La méthode des entretiens en autoconfrontation croisée leur donne l'occasion de revenir sur leur activité dans des contextes différents. Ils font alors l'expérience des possibilités de liaison et de déliaison de leur activité au sein du nouveau contexte dans lequel ils prennent part. Ces traversées sont de véritables expériences de transformation, de contradiction, de discordance, de dysharmonie durant lesquelles les internes se hissent, pour le dire à la manière de Vygotski, une tête au-dessus d'eux-mêmes (Vygotski, 1933/1978, p. 102). De fait, des tensions se constituent entre ce qui a été réalisé et ce qu'en dit l'interne en s'adressant au chercheur et/ou à son collègue. Les professionnels peuvent alors s'expliquer avec leur activité. Ils s'y réfèrent, non pas

11. Cet interne est également en dernière année d'internat et comme son collègue, il connaît l'hôpital où il sera affecté à la fonction de chef de clinique. 
pour la commenter de "l'extérieur », mais pour agir avec elle, au travers d'elle, sur elle, voire contre elle et au-delà d'elle. En fait, nous plaçons les professionnels moins en situation de nous expliquer leur activité que de s'expliquer « avec-sur-contre » leur activité. Ces mouvements qui déplacent le statut de l'expérience vécue leur fournissent l'occasion d'appréhender leur activité, de la saisir autrement, de la re-découvrir. Simultanément, ils permettent aux chercheurs de saisir les ressorts du développement de l'activité et ses empêchements.

\section{1.- De la confrontation aux images de l'activité à la confrontation aux gestes du « patron»}

Le premier passage est extrait de l'autoconfrontation simple de l'interne dont nous venons de décrire plus haut la situation de travail à laquelle il a pris part. Il commente son activité pour le chercheur, et il tente de justifier le peu d'énergie qu'il met à aspirer l'eau dans le ventricule gauche.
L'extrait débute alors que les images défilent et que l'interne commente son activité au chercheur. Par ailleurs, le nom du praticien hospitalier a été modifié pour conserver l'anonymat des professionnels.
1 - Interne 1 : J'aspire, là il veut le faire lui-même, ce n'était pas assez vigoureux je pense à son avis, donc il me prend l'aspiration des mains pour le faire lui-même
2 - Chercheur: On voit il le fait, et là, on te voit en position d'attente
3 - Interne 1: Oui, je laisse, je le laisse faire (5 secondes sans échanges verbaux)
4 - Interne 1: Là il calibre
5 - Chercheur: (il arrête les images) C'est pas assez vigoureux
6 - Interne 1: Manifestement non, mais Monsieur Dupond est parfois assez brutal entre guillemets comme chirurgien, c'est un chirurgien qui aime bien, donc euh, manifestement je n'aspirais pas de façon assez vigoureuse pour lui, donc c'est pour ça qu'il l'a fait lui-même
7 - Chercheur: Vigoureux, ça veut dire, il faut euh
8 - Interne 1: Aspirer vigoureusement, euh, sachant que ça ne modifie pas l'aspiration mais bon (rire) ou bien je n'étais pas assez rapide, assez vif pour lui, je ne sais pas, mais euh, pourtant je le sais en plus, là encore, c'est quelque chose que j'avais intégré, que je sais, et que lorsqu'il fait le geste, qu'il met de l'eau, il aime bien aspirer de façon très euh, alors je ne sais pas pourquoi je ne l'ai pas fait.

Dans le premier tour de parole de cet extrait, l'interne montre une certaine indifférence vis-à-vis des gestes : le sien, qu'il ne remet pas directement en cause; et celui du " patron », qu'il tente pratiquement de banaliser. À ce moment précis du dialogue, l'interne ne reprend pas son geste. Il n'en fait pas un objet de conversation. Il le laisse à distance d'une discussion potentielle, d'un approfondissement discursif. Mais il y a distance et distance. Celle-ci réalise probablement moins la marque d'un recul permettant de revenir sur les gestes qu'une non-différenciation avec les images, les émotions, les relations inter-individuelles. Autrement dit, cette mise à distance peut être le contraire d'une prise de distance réflexive. Nous y reviendrons.

Au tour de parole 2, nous tentons de poursuivre la discussion en revenant aux images de l'activité qui défilent (« et là, on te voit en position d'attente »), et en incitant l'interne à prolonger l'élaboration sur son activité, la re-prise de ses gestes.

Il répond par une réplique courte et directe: " je le laisse faire ». L'interne satisfait tout d'abord la demande du chercheur, puis après une pause de quelques secondes, il poursuit la conversation sur la base d'un autre objet: « Là il calibre » (tour de parole 4).

Au tour de parole 5, nous lui proposons de revenir sur la « vigueur» du geste. Cette demande d'explicitation, qui est aussi une tentative pour soutenir son activité de pensée sur le premier objet du dialogue - l'aspiration —, conduit l'interne à un début d'analyse du geste du senior.

Dans le tour de parole 6, l'interne qualifie ainsi de « brutal» le geste du chirurgien. En fait, il se saisit de notre demande d'explicitation pour critiquer le geste du senior tout en s'interrogeant sur sa propre activité d'aspiration (« manifestement je n'aspirais pas de façon assez vigoureuse pour lui »). 
En 7, nous réitérons notre proposition sur le geste insuffisamment vigoureux. Poussé par notre insistance et notre demande d'explicitation, l'interne débute son énoncé (tour de parole 8) par une nouvelle critique adressée à son « patron » : réaliser un geste vigoureux ne modifie pas vraiment l'efficacité de l'aspiration. Le rire qui suit le désaccord que l'interne manifeste avec le senior libère une énergie émotionnelle jusqu'alors retenue. Il nous renseigne aussi, et en partie, sur ce qui se joue dans l'activité de l'interne en autoconfrontation simple. Sans rechercher l'exhaustivité, nous pouvons noter que la fermeté du geste du « patron » de prise en main de la canule, la relative passivité à laquelle l'interne se confronte en situation d'intervention, les débats avortés sur les différentes manières de faire qui ne peuvent pas être menés directement avec sa hiérarchie ${ }^{12}$, forment un ensemble de conflits traversant l'objet même de l'activité d'aspiration. Ces conflits de l'activité re-pris, re-découverts, retrouvés ne laissent bien évidemment pas le sujet indifférent. En se confrontant aux images, l'interne éprouve, dans un autre contexte, les difficultés vécues pendant l'intervention. Et le rire, libérant une certaine tension, donne alors l'occasion à l'interne de se détacher des images de son activité pour poursuivre une activité de pensée sur son geste d'aspiration.

D'ailleurs, la suite de son énoncé est d'avantage tourné vers sa propre activité que vers celle du praticien hospitalier. Il s'engage ainsi dans une analyse de son activité qui lui permet de prendre la mesure d'une apparente contradiction inhérente à son activité. Il sait que le chirurgien va lui demander d'effectuer un geste vigoureux, et c'est pourtant en connaissance de cause qu'il n'exécute pas le geste attendu: « je le sais en plus [...] c'est quelque chose que j'avais intégré ». Dans le cours même de l'intervention chirurgicale, l'interne semble donc s'adresser au praticien hospitalier. Si celui-ci a le pouvoir de choisir les gestes opératoires adéquats ou non, l'interne peut répliquer par un geste dont il sait à l'avance qu'il ne conviendra pas. Malgré tout, cet ensemble contradictoire le laisse perplexe. Il termine son propos par une interrogation: « je ne sais pas pourquoi je ne l'ai pas fait».

Ce sentiment d'étonnement est pour nous un résultat très important. En devenant l'observateur extérieur de son activité d'aspiration, en s'appropriant le cadre dialogique de l'autoconfrontation simple, en re-découvrant son geste, l'interne questionne simultanément les manières de faire et de prendre les choses stabilisées dans le métier de chirurgien. Plus encore, en tentant de répondre à notre demande d'explicitation, l'interne convoque l'histoire collective de ce métier, au mieux, pour l'aider à se sortir de l'impasse du difficile à dire et à décrire, ou au contraire, pour le placer en situation désemparée face à ce qu'il se voit faire sur les images. Dans tous les cas, il éprouve, même en négatif, la fonction psychologique du collectif: une ressource pour l'activité personnelle.

Nous faisons de ce prologue, avec ses points d'achoppement, ses limites et ses résidus dialogiques (Scheller, 2001), une phase essentielle de la poursuite du dialogue en autoconfrontation croisée.

\section{2.- De la confrontation aux gestes du « patron » à la confrontation à sa propre activité à-venir}

Lors de l'autoconfrontation croisée ${ }^{13}$, les deux internes, assis côte à côte et face au moniteur, observent les images de l'activité que nous avons décrites plus haut. Au moment où le praticien hospitalier ôte la canule des mains de l'interne, ils se mettent à sourire, et l'interne 1 arrête le défilement.

Interne $1: 1$ 'interne ayant participé à l'activité professionnelle précédemment décrite

Interne 2: collègue du même niveau d'expertise ayant lui-même réalisé une autoconfrontation simple sur la base des images de sa propre activité

$[\ldots]$ : marque une coupure que nous avons réalisée dans cette présentation afin de réduire la longueur de l'extrait

1 - Interne 1 : Et vous lavez comme ça à grandes eaux, comme ils font là, l'aorte là

12. Dans le cadre de notre étude, nous avons pu observer que les internes n'avaient que de trop rares occasions de discuter des manières de faire: de rares discussions entre internes, pratiquement aucuns débats avec les praticiens hospitaliers et encore moins avec le chef de service.

13. Au moment de cette autoconfrontation croisée, les deux internes font référence à leur service d'origine dans lequel ils savent également qu'ils retourneront effectuer leur clinicat. 
2 - Interne 2: On lave avec des seringues

3 - Interne 1: Ah vous lavez vous

4 - Interne 2 : Avec des seringues [...] nous on lave deux fois et puis voilà $[\ldots]$

5 - Chercheur: Et là du coup, tu (adressé à Interne 1) es en position d'observer, de regarder comment il fait pour

6 - Interne 1: Oui, là il opère tout seul là

7 - Interne 2: Oui mais en même temps, je pense qu'il regarde comment Dupond fait

8 - Interne 1 : Mouais, je pense que ça, ça sert à rien, mais bon

9 - Interne 2: De quoi, d'aspirer?

10 - Interne 1 : Et bien, je ne sais pas moi

11 - Interne 2: Non mais les petites calcifications, il faut les enlever

12 - Interne 1 : Oui tu les enlèves, mais parce que tu crois qui y'en a qui tombent beaucoup

13 - Interne 2 : Ah des fois y'en a qui tombent hein [...] ah mais toi qu'est-ce tu dis, de laver ça ne sert pas?

14 - Interne $1:$ Ah ça aussi je pense que ça ne sert à rien non

15 - Interne 2 : Ah non, moi je pense que ça sert

16 - Interne 1 : Sauf si tu vois des choses qui tombent mais pas de façon systématique, mais j'ai peut-être tort

17 - Interne 2: Moi ça me plaît assez de laver, nous on lave pas mal, on met deux grosses seringues et on aspire.

Les sourires simultanés des deux internes nous renseignent sur les partages d'implicites à ce moment de l'autoconfrontation croisée. Mais au lieu de discuter du geste ferme du « patron », l'interne 1 préfère, en s'adressant à son collègue, interroger la pertinence du geste chirurgical: laver le ventricule gauche « à grandes eaux » (tour de parole 1). La réponse de l'interne 2 (tour de parole 2) renvoie aux manières de faire du service qui l'a formé et dans lequel il va exercer la fonction de chef de clinique. Il indique qu'il utilise des seringues plutôt qu'une coupelle pour verser l'eau dans le ventricule. Dans le troisième tour de parole, l'interne 1 réitère son interrogation. Son collègue répond en déployant légèrement le protocole qu'il réalise dans ces conditions. En utilisant un « on » générique, il précise que le lavage s'effectue deux fois de suite. Ici encore, la réponse se construit sur une évidence: " on lave deux fois et puis voilà » (tour de parole 4).

En essayant de revenir sur les images gelées par la position pause du magnétoscope, le chercheur tente de " rafraîchir » la situation conversationnelle. Il rompt avec l'objet de l'interaction verbale pour mieux revenir à l'activité réalisée. L'interne 1 répond partiellement à la question du chercheur puisqu'il n'interroge pas directement son activité mais celle du " patron ». C'est d'ailleurs son pair qui répond à sa place: « je pense qu'il regarde comment Dupond fait » (tour de parole 7). Cette réponse conduit les deux internes à une suite d'échanges construits sur des implicites non partagés et produit ainsi une suite d'incompréhensions.

Tout d'abord, l'interne 2 questionne son collègue sur ce qui ne sert à rien (tour de parole 9) et affirme, après une réponse évasive de l'interne 1 (tour de parole 10), toute l'importance qu'il y a à prélever les débris calciques éventuellement tombés dans le ventricule gauche ( « il faut les enlever », tour de parole 11). En s'appuyant sur les échanges précédents, l'interne 1 revient sur son affirmation du tour de parole 8 et précise la prémisse sous-jacente à son propos : «parce que tu crois qui y’en a (des débris calciques) qui tombent beaucoup », tour de parole 12). La réalisation de cette proposition installe un certain déséquilibre dans l'interaction. L'interne 2 ne comprend plus alors très bien, à ce moment de l'interaction, ce que son collègue veut dire: « ah mais toi qu'est-ce tu dis, de laver ça ne sert pas? » (tour de parole 13). La réponse de l'interne 1 est très claire. Il considère que laver le ventricule gauche ne sert à rien (tour de parole 14).

Une controverse s'engage alors entre les deux internes. Pour l'interne 2, laver le ventricule gauche est au contraire un geste opératoire nécessaire (tour de parole 15). Cette affirmation pousse alors l'interne 1 à poursuivre l'échange en essayant de trouver des arguments. Il interroge le caractère systématique d'une telle action. Le lavage ne serait primordial que si le chirurgien aperçoit des dé- 
bris dans le ventricule. En affirmant que certains gestes « ne servent à rien », il dépasse largement les limites qu'il ne s'autorise pas à franchir dans l'exercice de son travail quotidien. D'ailleurs et simultanément, il prend des distances vis-à-vis de sa propre proposition : finalement, il a peut-être tort (tour de parole 16). Ce faisant, il est confronté aux dilemmes de l'activité qui sera la sienne lorsqu'il sera chef de clinique. De son côté, l'interne 2 poursuit la controverse, et affiche une nouvelle fois une certaine détermination associée à une tranquillité. Il colore affectivement le geste de lavage (« ça me plaît assez », tour de parole 17), puis il utilise une nouvelle fois le « on » pour intégrer son argument dans une manière de faire partagée et routinisée (« nous on lave pas mal », ibidem).

Comment comprendre et expliquer les prises de position distinctes des deux internes lorsqu'ils s'expliquent avec le geste opératoire consistant à laver le ventricule gauche? Que réalisent-ils l'un et l'autre dans cette conversation? Quel est le rôle et la fonction psychologique du collectif dans cet extrait?

\section{3.- Le genre professionnel: un moyen d'action pour les internes}

Pour tenter de répondre à ces questions, nous souhaitons reprendre certains résultats — en ligne directe avec la problématique du collectif — issus de travaux en ergonomie et en psychologie du travail dans le champ de l'hôpital et revenir sur les spécificités de l'activité des internes dans ce service de chirurgie cardiaque.

Tout d'abord, et comme l'a montré Theureau (1981), la coordination temporelle des activités des différents professionnels subit des aléas constants. Les tâches sont très souvent reprogrammées et les internes qui arrivent dans un service doivent s'orienter dans un labyrinthe d'un monde professionnel déjà fabriqué. Les fonctionnements quotidiens des professionnels s'inscrivent dans des histoires qui peuvent être énigmatiques pour un interne en début de stage dans un service. Par exemple, les activités de diagnostic et d'anticipation pendant une intervention chirurgicale sont parfois en désynchronisation avec l'objet de travail (Hoc, Amalberti, Cellier, \& Grosjean, 2004). Les ressorts de cette régulation le plus souvent implicite se construisent dans l'histoire des services. D'ailleurs, les services peuvent s'apparenter à des tours de contrôle éclatées (Martin, \& Gadbois, 2004) dont la fonction est de proposer aux soignants des informations pertinentes en relation avec le cours du travail collectif.

De notre côté, le dispositif méthodologique que nous avons mis en place, avec la méthode des entretiens en autoconfrontation croisée qui le réalise, nous donne l'occasion de revenir sur ces travaux et de réinterpréter ces résultats. En nous focalisant sur la dimension collective de l'activité des internes, nous voudrions insister sur certaines conditions du développement de l'activité et simultanément de ses empêchements.

S'il fallait synthétiser ce que nous retenons pour cet article des travaux que nous avons mentionnés, nous pouvons, avec l'aide de Bruner (1996, p. 188), dire que les internes doivent déchiffrer, indépendamment de leur niveau d'expertise, un véritable « rébus » complexe où se mêlent des manières de faire, de dire, de prendre les choses particulières du service. En somme, avant de développer des habiletés manuelles, avant d'acquérir des gestes de métier, avant d'aiguiser leur sens clinique, les internes doivent apprendre à s'orienter dans un champ de contraintes et d'énigmes. De la préparation des interventions jusqu'à la sortie du patient, les internes s'insèrent dans une histoire professionnelle sédimentée dans des manières de faire, des manières de prendre les choses, des manières de penser.

Pour illustrer cette « immersion » des internes, l'installation du patient sur la table d'opération peut nous aider à prendre la mesure du poids de l'histoire. Dans le service de chirurgie cardiaque où nous avons réalisé notre étude, cette installation obéit à des routines précises qu'il convient d'apprendre. Comme les champs disposés sur le corps du malade ont fait l'objet d'un paramétrage spécial, les internes doivent imiter les gestes du chef de clinique. Le binôme de travail — interne et chef de clinique - se dispose en face à face, les champs entre les mains, la table d'opération et le malade entre eux. Ce qui est demandé à l'interne n'est pas seulement de réaliser simultanément et avec exactitude 
les gestes qu'il voit faire, il doit aussi comprendre les intentions qui sous-tendent les actions. Sans cette compréhension, les internes ne peuvent réellement construire, étayer et utiliser leurs habiletés. Dans la salle d'opération, l'anticipation de ce qu'il y a à faire est l'une des clés de l'efficacité (Clot, Doray, \& Michel, 1992). Comprendre est alors l'une des ressources de l'action des internes.

Mais lorsque les tâches ne relèvent plus de routines partagées dans ses moindres détails, les internes doivent mémoriser les gestes réalisés par les différents chirurgiens - seniors, chefs de clinique. Mieux, ils doivent apprendre à aider en fonction des habitudes des uns et des autres. Comprendre devient ainsi plus compliqué. L'interne doit, à l'instar d'autres novices, à la fois tenter de comprendre, et accepter de ne pas comprendre (Amalberti, 1996). Dès lors, comment appréhender les différentes manières de faire? Comment se saisir de ces différences pour en faire quelque chose? Quel statut psychologique ont-elles pour l'interne? Comment s'en débrouillent-ils?

Les internes peuvent, au cours d'une même matinée, travailler comme aide avec deux opérateurs différents usant de techniques distinctes. Face à ces exigences, les internes doivent malgré tout répondre aux attentes des opérateurs: aider efficacement. Ce faisant, ils apprennent à observer dans le détail les gestes des chirurgiens.

Dans le cours de l'autoconfrontation croisée avec les deux internes, l'un d'eux, à la suite d'une demande d'explicitation du chercheur, a précisé comment il conçoit ce rôle d'aide dans ces contextes toujours spécifiques:

Interne 1: «Dès qu'on voit qu'il (l'opérateur) amorce un geste, on dit d'accord, lui il choisit ce chemin là, donc tout de suite on répond dans le sens où il veut aller quoi, c'est sous-cortical, c'est bulbaire (rire) » (extrait de l'autoconfrontation croisée entre l'interne 1 et 2).

Cette capacité à s'ajuster aux gestes de l'opérateur demande un haut niveau d'appropriation des habitudes et des différentes manières de faire. C'est parce que les internes connaissent les principales manières de faire et ses variantes, qu'ils peuvent alors être disponibles pour se laisser guider par les gestes de l'opérateur principal et s'y ajuster. Si les gestes de l'opérateur principal « stimulent » l'interne au "niveau sous-cortical », si les internes réagissent de manière automatique, c'est parce certains gestes fonctionnent comme des véritables mots de passe que seuls certains professionnels de ce service savent décrypter.

Dans les travaux effectués en clinique de l'activité, nous avons nommé genre professionnel (Clot, 1999) la partie sous-entendue de l'activité appropriée par les professionnels. Le genre est ce que les professionnels connaissent, reconnaissent, comprennent, estiment, jaugent, évaluent, ressentent ou redoutent dans les conditions concrètes de l'exercice de leur travail. Il est donc à la fois le produit d'une histoire collective et un instrument de l'action individuelle. Autrement dit, les manières de faire des chirurgiens cardiaques appartiennent à une histoire collective dans laquelle se " glissent » des internes pour tenter d'agir efficacement.

Tel que nous le concevons, le geste de métier des chirurgiens est ainsi réglé de l'extérieur par le genre professionnel. Les internes apprennent non seulement des gestes opératoires, ils doivent également faire leur une histoire. C'est en traversant des situations diversifiées et des contextes dans lesquels des gestes professionnels différents sont réalisés par des opérateurs singuliers que les internes éprouvent le genre et ses variantes. Plus encore, c'est en s'essayant à la réalisation de certains gestes - sous la responsabilité de l'opérateur principal — qu'ils pourront disposer, pour eux-mêmes, du genre. En s'immisçant dans le genre, en le faisant sien, les internes peuvent alors l'utiliser comme ressource afin d'agir efficacement.

\section{4.- Observer et imiter: un moyen d'appropriation du genre professionnel}

Dans ces conditions, les gestes opératoires, que les internes effectuent sous le contrôle de l'opérateur 
principal, imitent ceux des chirurgiens plus expérimentés. En nous appuyant sur le travail de Wallon, nous souhaitons insister sur la complexité de ce phénomène.

Pour cet auteur, l'imitation ne se réalise que par une sorte d'assimilation de soi à l'autre qui captive l'intérêt de l'observateur (Wallon, 1942/1970). L'apprentissage par imitation pourrait donc bien correspondre à un dialogue sensori-moteur dans lequel les interactants tentent d'adopter le point de vue de l'autre à partir de ce qu'ils observent conjointement (Tomás, Simonet, Clot, \& Fernandez, à paraître). L'observation des gestes du chirurgien expérimenté est ainsi accompagnée d'intentions " qui imprègnent nos centres moteurs comme une sorte de méditation, et qui, passée la période nécessaire d'incubation, vont donner lieu aux gestes correspondants [...]. L'apprenti chirurgien qui n'a fait longtemps qu'assister son maitre sait à l'occasion déployer une dextérité dont ses propres essais ne peuvent suffire à rendre compte » (Wallon, 1943/1990, p. 108-109).

Mais l'imitation n'est pas seulement fusion et participation. Elle est aussi « dédoublement de l'acte à exécuter d'avec le modèle » (Wallon, 1942/1970, p. 144). S'approprier le genre professionnel, avoir du métier, accomplir de beaux gestes supposent simultanément de s'affranchir du travail des autres, de se différencier des manières de faire d'autrui. De fait, l'appropriation d'un geste de métier serait alors la capacité de changer de perspective (Tomasello, 2004), c'est-à-dire d'emprunter un geste étranger en adoptant les intentions d'autrui avant de soumettre le geste à ses propres intentions réalisées dans l'action. Dans le cas des internes, ce passage d'une perspective à une autre est l'une des caractéristiques des stages d'internat. En effet, les internes peuvent comparer les différents gestes entre eux en puisant dans leurs multiples expériences. Cette comparaison qui s'étale dans des temps synchroniques et diachroniques leur donne l'occasion de repérer " l'autre geste dans le même ». C'est par un jeu de comparaison que les internes peuvent se défaire des gestes d'autrui en passant d'un service à un autre, d'une salle à une autre, d'un opérateur principal à un autre. Le " même geste » peut alors se décanter, se détacher. Le geste devient disponible et appropriable non seulement par l'interne, mais aussi et surtout pour l'interne, pour la réalisation de gestes opératoires efficaces dans une situation donnée.

Revenons maintenant aux dialogues, réalisés en autoconfrontation croisée, entre les deux internes. Leur conversation porte sur un geste professionnel - laver le ventricule gauche - qui semble être suffisamment approprié pour être critiqué. En effet, dans les deux cas, ils possèdent une distance suffisante vis-à-vis de ce geste pour tenter de l'évaluer, de le discuter, de le critiquer, de l'analyser. Ils se sont appropriés un geste communément accompli et ils peuvent se risquer à le discuter, voire à l'« attaquer ».

Le geste discuté par les internes est un geste qui s'inscrit dans l'histoire du métier, dans celle des développements techniques et technologiques (Cabrol, 2006), dans celle des manières de faire génériquement admises par les professionnels. Il cristallise en quelque sorte une partie de cette histoire collective. Et ce que nous voyons fonctionner dans et entre les échanges des deux internes extraits de l'autoconfrontation croisée, c'est probablement moins une discussion directement engagée sur l'objet que leur rapport avec l'histoire collective, avec le genre professionnel.

\section{5.- Le geste collectif et le collectif de geste}

La transmission des gestes professionnels ne se réduit pas à un transfert linéaire, du praticien expérimenté au novice. Le compagnonnage qui caractérise l'une des trames de la transmission professionnelle du métier de chirurgien contraint l'opérateur à adresser son geste à l'interne qui le seconde. Les gestes opératoires réalisés dans ces conditions sont donc toujours multi-adressés. Ils sont à la fois dirigés vers l'objet de l'activité, dans la situation que nous étudions, il s'agit du remplacement d'une valve aortique, et vers les autres professionnels dont l'activité porte sur cet objet, c'est-à-dire le chef de clinique, l'interne, l'instrumentiste et dans une certaine mesure, l'anesthésiste, l'infirmier anesthésiste et le perfusionniste. Le geste d'aspiration est donc un geste collectif, qui s'inscrit dans une activité collective, et qui s'appuie sur des routines, des formalisations, une histoire professionnelle. 
D'ailleurs, il n'est pas rare d'entendre dans ce type de service qu' " à technique égale, c'est l'équipe qui fait la différence ». On peut alors concevoir l'effectuation d'un geste comme le résultat d'une condensation de l'activité collective: à la fois de l'histoire collective constamment retouchée dans l'exercice du travail et des relations intra et inter-professionnelles au moment de la réalisation du geste. De fait, le geste n'est jamais totalement « collectif», il porte en lui l'empreinte des collectifs — passé, à venir et présent —, mais il est toujours irréductiblement individuel, c'est-à-dire réalisé et parfois stylisé par un professionnel singulier. On peut dire que le collectif borde le geste en lui procurant une contenance.

Du côté de l'activité de l'interne, celui-ci doit pouvoir, comme nous l'avons entrevu, à la fois ajuster ses gestes au prototype à imiter et se dissocier du geste d'autrui. Son activité doit pouvoir se frayer un chemin dans une histoire de métier, mais aussi à contre-courant de celle-ci. L'activité de l'interne s'immisce dans une histoire qui ne peut partager et utiliser comme instrument de son action individuelle que par un mouvement de dissociation, de prise de distance.

De fait, le geste modèle, dans le cours de l'apprentissage, peut être objet d'une délibération au sein d'une activité de pensée intérieure. Au mieux, cette délibération peut aussi se réaliser dans des situations d'échanges et de discussions entre professionnels. Ce faisant, après « une phase alternée de participation sensori-posturale avec autrui et d'élaboration posturo-gestuelle » (Wallon, 1942/1970, p. 151), le geste est comparé avec ceux réalisés ailleurs, avec celui que l'interne réaliserait s'il était à la place du premier opérateur, avec celui que le chirurgien aurait pu faire s'il avait choisi une autre technique opératoire. Autrement dit, les gestes des chirurgiens expérimentés n'ont pas toujours la même fonction psychologique pour les internes.

$\mathrm{Ce}$ « collectif de geste », d'abord énigme et modèle à reproduire, peut devenir un moyen de développement de l'activité individuelle. Par les tentatives de prendre part à cette histoire, à ce collectif de geste, en les faisant siens tout en les déplaçant, l'activité des internes prend sa source dans ces gestes à imiter avant que ces derniers ne deviennent, dans le meilleur des cas, des ressources pour le développement de leurs propres gestes. À concevoir ainsi le collectif de geste, on peut alors comprendre comment « le collectif est simultanément à l'intérieur de l'individu et qu'il s'y développe en fonction des échanges entre "gens du métier" »(Clot, 2003, p. 39), novice compris ${ }^{14}$. Mais le collectif n'est jamais totalement à l'intérieur d'un professionnel. Le collectif déborde toujours l'activité individuelle, ce qui permet aux internes, non seulement d'éprouver, dans l'exercice de leur fonction ce qu'il y a de plus en eux, mais aussi ce qu'il y a de plus en l'autre, notamment les chirurgiens expérimentés.

Les internes ont alors du métier lorsque la fonction psychologique du collectif de geste habite leur activité. La traversée des différents contextes de travail, avec les manières de faire spécifiques à chaque praticien hospitalier, leur fournit l'occasion de reconnaitre la trace du collectif dans les gestes individuels des opérateurs. Et c'est en se confrontant autant de fois que nécessaire avec les différents gestes de métier, que les internes peuvent réussir à acquérir une sorte d'équilibre, produit du rapport entre le collectif et leur activité, leur permettant de mettre à distance d'eux-mêmes et des autres, les gestes opératoires. Dans ce cas, l'interne peut se reconnaître dans le collectif, dans le geste collectif, et il peut alors s'expliquer avec le collectif de geste.

\section{6.- Conclusion}

Suivre l'activité conversationnelle de l'interne 1, aussi bien pendant l'autoconfrontation simple et l'autoconfrontation croisée, nous permet de saisir, à partir de ses réalisations langagières, les dissonances à l'œuvre dans son activité de pensée. Dans l'extrait de l'autoconfrontation simple, et sur la base des images de son activité filmée, l'interne ébauche une première critique du geste du " patron » avant de reprendre sa propre activité, et de convenir qu'il ne sait pas exactement pourquoi il fait ce

14. Comme le travail des chirurgiens est souvent comparé à celui des pilotes d'avion, nous signalons le travail de Caroline Moricot (2007) qui éclaire cette position du collectif dans l'individu à partir de l'engagement du corps des pilotes de ligne des avions automatisés. 
qu'il fait. En revenant sur les mêmes images, la discussion des deux internes, pendant l'autoconfrontation croisée, permet à l'interne 1 d'exposer son point de vue et de s'exposer au métier, aux manières de faire stabilisées.

Les tours de parole de 13 à 17 concrétisent les différents points de vue. L'interne 2 ne conçoit pas la dissection d'une valve aortique sans laver le ventricule gauche afin de retirer les débris calciques. Or son collègue pense que cela ne sert à rien. Mais dans le même temps, il relativise sa position: il convient qu'il a peut-être tort.

Mieux, si l'on reprend ce qu'il dit concernant la réaction « bulbaire » que les internes doivent avoir intériorisé afin de répondre adéquatement aux attentes de l'opérateur principal, on ne peut être qu'interpellé par cet ensemble contradictoire. L'interne 1 semble travaillé par quelque chose qui lui échappe et qu'il teinte parfois d'une émotion de défi vis-à-vis de l'autorité représentée par le " patron ». Ce quelque chose qui lui échappe semble au contraire être utilisé par l'interne 2. C'est avec une certaine tranquillité qu'il ancre son point de vue dans la controverse en cours. D'ailleurs il n'hésite pas à teinter également son discours d'une coloration affective (" moi ça me plaît assez de laver », tour de parole 17).

Pour nous, et cela reste à étayer par d'autres travaux en cours ${ }^{15}$, c'est la fonction psychologique de collectif qui est travaillée et mise au travail par les deux internes. En s'engageant dans une controverse sur des gestes professionnels, les internes s'appuient sur l'histoire du collectif, le geste collectif et le collectif de geste. Mais dans le cours de leur conversation, ces répondants les secondent différemment. Autant l'interne 1 est travaillé par le geste collectif, qu'il essaie de remettre en question, autant l'interne 2 met au travail le collectif de geste, c'est-à-dire la part collective du geste appropriée par et pour lui.

Plus exactement, ce que nous voyons à l'œuvre dans le développement de l'activité de pensée de l'interne 1, c'est le travail de dysharmonie entre son activité et son milieu. Pour tenter de prendre une place dans un milieu de travail possédant une histoire collective fortement élaborée, l'interne 1 semble construire une sorte de déphasage, dont la fonction psychologique ne peut se comprendre qu'à l'aune du rapport au collectif. Si, comme aime à le penser Canguilhem (1965/1992), « le propre du vivant, c'est de se faire son milieu, de se composer son milieu » (p. 143), l'interne 1 tente de se construire un milieu en remettant en cause le répondant collectif. La controverse avec son collègue réalise ainsi une épreuve le reliant aux autres chirurgiens en passant par l'histoire collective.

Pour le dire autrement, les dialogues que nous avons organisés à partir des images de son activité le poussent à analyser la non-coïncidence entre ce qu'il fait, ce qu'il ferait, ce qu'il fera et ce que son « patron » réalise, mais aussi, et c'est pour nous le plus important, les dialogues lui permettent d'éprouver le disparate qu'il trouve en lui, dans ses rapports avec ce milieu. Cette épreuve n'est d'ailleurs pas exempte d'affect ${ }^{16}$. C'est parce que l'interne est affecté par le dysharmonique, l'inadéquation, l'inachèvement que le dialogue avec son collègue lui donne aussi l'occasion de se risquer à chercher en lui et dans son milieu de travail des gestes alternatifs.

À ne considérer que l'activité dialogique, il nous semble donc que l'un des moteurs de la conversation entre ces deux internes est moins le collectif en soi, que le rapport entre ce qu'il y a de plus chez l'autre et ce qu'il y a de plus en soi. Ce seraient alors les excédents que nous possédons par la médiation du collectif qui détermineraient la motricité dialogique et les possibilités de développement de l'activité de pensée.

Nous voulons terminer cet article en avançant quelques directions de recherche. En situation de travail et face à la tâche demandée par le praticien hospitalier, l'interne 1 se démène entre la surabondance fonctionnelle qu'il a acquise par ses multiples expériences et son rapport au geste réalisé

15. Les séminaires de recherche du groupe Geste Automatisme Mouvement (GAM) de l'équipe clinique de l'activité tentent, sur la base notamment de matériaux empiriques, d'approfondir les rapports entre le développement des gestes et le collectif de travail.

16. Nous voulons signifier ici la dette que nous avons à l'égard du travail de Simondon, notamment les quelques pages qu'il a consacré à l'angoisse dans son ouvrage L'individuation psychique et collective, 1989, Aubier. 
par le praticien qui lui fait face. Ensuite, et dans le cours de l'autoconfrontation croisée, il éprouve ce que son collègue fait du collectif de geste. L'interne 1 fait alors par deux fois l'expérience de ce qu'il y a de plus que lui en soi par la médiation de ce qu'il y a de plus dans l'autre. Dans ces deux contextes, il est affecté par un excès qu'il porte en lui, et dans lesquels il se démène, mais qui n'est pas directement lui. Les gestes professionnels, l'histoire collective et les affects entretiendraient alors des liens qu'il conviendrait d'instruire non seulement pour analyser le développement de l'activité de pensée dans le cours des autoconfrontations croisées mais aussi pour examiner de façon systématique le problème du développement du geste et de son appropriation en situation de travail.

\section{BIBLIOGRAPHIE}

Amalberti, R. (1996). La conduite des systèmes à risques. Paris: PUF.

Bruner, J. (1996). L'éducation, entrée dans la culture. Paris: Éditions Retz.

Cabrol, C. (2006). De tout coeur. La nouvelle chirurgie cardiaque. Paris: Odile Jacob.

Canguilhem, G. (1965/1992). La connaissance de la vie. Paris: Vrin.

Clot, Y. (1999). La fonction psychologique du travail. Paris: PUF.

Clot, Y. (2003). Le collectif dans l'individu ? In G. Vallery, \& R. Amalberti (Eds), Modèles et pratiques de l'analyse du travail. 1988-2003, 15 ans d'évolution (pp. 33-43). Paris: Actes du XXXVIIIème Congrès de la SELF.

Clot, Y. (2004). Le travail entre fonctionnement et développement. Bulletin de psychologie, 57 (1), 5-12.

Clot, Y., Doray, B., \& Michel, A. (1992). Efficacité dans les services. Rapport pour le Ministère de la Recherche.

Clot, Y., Faïta, D., Fernandez, G., \& Scheller, L. (2001). Entretiens en autoconfrontation croisée : une méthode en clinique de l'activité. Éducation Permanente, 146 (1), 17-25.

Hoc, J.-M., Amalberti, R., Cellier, J.-M., \& Grosjean, V. (2004). Adaptation et gestion des risques en situation dynamique. In J.-M. Hoc, \& F. Darses (Eds.), Psychologie ergonomique: tendances actuelles (pp. 15-48). Paris: PUF.

Martin, C., \& Gadbois, C. (2004). L'ergonomie à l'hôpital. In P. Falzon (Sous la dir.), Ergonomie. Paris: PUF.

Moricot, C. (2007). L'irréductible engagement du corps. Le cas des avions automatisés. Communications, 81, 201-213.

Scheller, L. (2001). L'expérience du travail dans le cadre dialogique. Travailler, 6, 71-88.

Theureau, J. (1981). Analyse ergonomique de l'espace de travail et programmation des nouvelles unités de soins hospitalières. Soins, 26 (12).

Tomás, J.-L., Simonet, P., Clot, Y., \& Fernandez, G. (à paraître). Le corps : l'œuvre du collectif de travail. Revue Corps.

Tomasello, M. (2004). Aux origines de la cognition humaine. Paris : Éditions RETZ.

Vygotski, L. (1931/1978). Problems of Methods. In L.S. Vygotsky, Mind in Society. The Development of Higher Psychological Processes (pp. 58-75). Cambridge, MA: Harvard University Press.

Vygotski, L. (1933/1978). The Role of Play in Development. In L.S. Vygotsky, Mind in Society. The Development of Higher Psychological Processes (pp. 92-104). Cambridge, MA: Harvard University Press.

Wallon, H. (1942/1970). De l'acte à la pensée. Paris: Flammarion.

Wallon, H. (1943/1990). La sensibilité affective: moi et non-moi. In H. Wallon, Écrits de 1926 à 1961, psychologie et dialectique (pp. 98-109). Paris: Messidor/Éditions sociales. 


\section{RÉSUMÉ}

L'objectif de cet article est de montrer comment la réalisation des gestes professionnels peut être étayée par l'histoire du métier et par les différentes manières de faire qui sont en usage dans le milieu professionnel. L'étude que nous avons menée dans un service de chirurgie cardiaque nous permet de saisir l'une des fonctions de cette histoire collective, et de l'évaluation des gestes par le collectif de travail : une ressource pour l'action individuelle. Le cadre méthodologique et théorique que nous avons mobilisé est celui de la clinique de l'activité. Nous avons ainsi réalisé deux films d'activité de deux internes volontaires en situation de travail lors d'une intervention chirurgicale. Ces enregistrements ont été suivis par des entretiens en autoconfrontions simple et croisée. Sur la base de deux extraits de ces entretiens, nous tentons de repérer les ressources individuelles permettant de soutenir l'activité dialogique dirigée vers un geste précis de travail. Ce faisant, nous interrogeons alors la fonction psychologique du collectif au regard des controverses professionnelles provoquées par la situation d'autocofrontation croisée. Plus encore, nous tentons de différencier le geste collectif du collectif de geste comme soutien de l'activité dialogique.

\section{MoTS CLÉS}

Geste, Geste collectif, Collectif de geste, Autoconfrontation croisée, Activité de pensée, Développement

\section{RESUMEN}

Explicarse con el colectivo del gesto: El caso de dos internos de cirugía cardiaca. El objetivo de este artículo es mostrar cómo la realización de los gestos profesionales puede estar sostenida por la historia del oficio y por las diferentes maneras de hacer que son de uso en el medio hospitalario. El estudio que hemos llevado a cabo en un servicio de cirugía cardíaca nos permite comprender una de las funciones de esta historia colectiva y de la evaluación de los gestos, por parte del colectivo de trabajo: un recurso para la acción individual. El marco teórico y metodológico que hemos utilizado es el de la clínica de la actividad. Hemos realizado así dos filmes de la actividad de dos internos voluntarios en situación de trabajo durante una intervención quirúrgica. Estas filmaciones han sido objeto de entrevistas de autoconfrontación simple y cruzada. Sobre la base de dos extractos de estas entrevistas, intentamos localizar los recursos individuales que permiten sostener la actividad dialógica dirigida hacia un gesto preciso de trabajo. Procediendo de esta manera, interrogamos entonces la función psicológica del colectivo respecto de las controversias profesionales provocadas por la situación de autoconfrontación cruzada. Mas aún, intentamos diferenciar el « gesto colectivo » del « colectivo del gesto » como sostén de la actividad dialógica.

Palabras clave

Gesto, Gesto colectivo, Colectivo del gesto, autoconfrontación cruzada, Actividad de pensamiento, desarrollo

\section{RÉFÉRENCEMENT}

Tomás J.-L. (2008). S'expliquer avec le collectif de geste : le cas de deux internes en chirurgie cardiaque. Activités, 5 (2) pp. 39-51, http://www.activites.org/v5n2/v5n2.pdf

Article soumis le 11 février 2008, accepté pour publication le 17 septembre 2008. 